\title{
eJustice in der Schweiz
}

\section{Projekt Justitia 4.0 aus der Perspektive von Richtern, Justizmanagern und IT-Verantwortlichen}

Das Hauptziel des Forschungsprojekts, das dieser Publikation zu Grunde liegt, besteht darin, die Erfahrungen und Strategien zu beschreiben, die im Rahmen verschiedener Innovationen im Bereich digitalisierter Gerichte (E-Gerichte) angewandt werden. Die hier vorgestellten Ergebnisse beinhalten den schweizerischen Teil eines transnationalen Projekts, an dem Brasilien und die Schweiz beteiligt sind. Die Forschung ist qualitativ und deskriptiv. Es wurden 22 ausführliche Interviews auf der Basis von teilstrukturierten Scripts mit Richtern, IT-Managern und Justizmanagern geführt. Die Auswertung der Ergebnisse gibt Aufschluss über die zentralen Triebkräfte und Hindernisse bei der Einführung von E-Gerichten im Schweizer Kontext.

Beitragsart: Science

Zitiervorschlag: Marcos de Moraes Sousa, eJustice in der Schweiz, in: «Justice - Justiz - Giustizia» $2020 / 4$ 


\section{Inhaltsübersicht}

1. Einführung

1.1. Ausgangslage und Kontext

1.2. Fokus der Studie

2. Hintergrund: Innovation im öffentlichen Sektor

3. Methodologie

4. Ergebnisse und Diskussion

5. Fazit und Anmerkungen

\section{Einführung}

\subsection{Ausgangslage und Kontext}

[1] Gerichtsmanagement hat sowohl politisch und administrativ als auch unter dem Gesichtspunkt der Kontrolle an Bedeutung gewonnen. ${ }^{1}$ Des Weiteren hat es sich im Bereich der öffentlichen Verwaltung als Forschungsgebiet etabliert. ${ }^{2}$ Vom Aspekt der Ressourcenverwaltung aus gesehen beinhaltet es auch die Verwaltung von Technologien. ${ }^{3}$ In dieser Hinsicht gilt Innovation als eines der zentralen Themen in der Forschung im Bereich der Gerichtsverwaltung, ${ }^{4}$ und die Anwendung von Technologien an Gerichten hat in den letzten Jahrzehnten die Aufmerksamkeit sowohl aus der Sicht von Fachleuten als auch aus der akademischen Welt auf sich gezogen. ${ }^{5}$

[2] Innovation bezieht sich auf die Einführung und Verbreitung neuer und fortschrittlicherer Produkte und Prozesse. ${ }^{6}$ Eine Kombination von Ressourcen und Kompetenzen kann für eine gegebene Organisation neu sein, sie kann jedoch auch von anderen Organisationen entwickelt worden sein; sie kann das Ergebnis interner Beschlüsse oder Folge der vom Umfeld bedingten Massnahmen sein. ${ }^{7}$

[3] Gerichtsinnovationen wurden in den organisatorisch-administrativen, politisch-rechtlichen und technologischen Dimensionen erforscht. ${ }^{8}$ Im technologischen Bereich liegt der Schwerpunkt bei den Informations- und Kommunikationstechnologien ICTs wie z.B.: die Nutzung des Internets durch Gerichte, ${ }^{9}$ das Verhältnis von Regierung zu Regierung, ${ }^{10}$ Computer-Tools, ${ }^{11}$ elektronische Rechtsverfahren, ${ }^{12}$ Einsatz künstlicher Intelligenz ${ }^{13}$ und andere Technologien.

[4] In der Schweiz ist die Zahl der vollständig in elektronischem Format eingereichten Fälle noch gering, jedoch haben die kantonalen und eidgenössischen Gerichte 2016 ein gemeinsames Projekt namens Justitia 4.0 initiiert, «to introduce electronic communication between judicial authorities

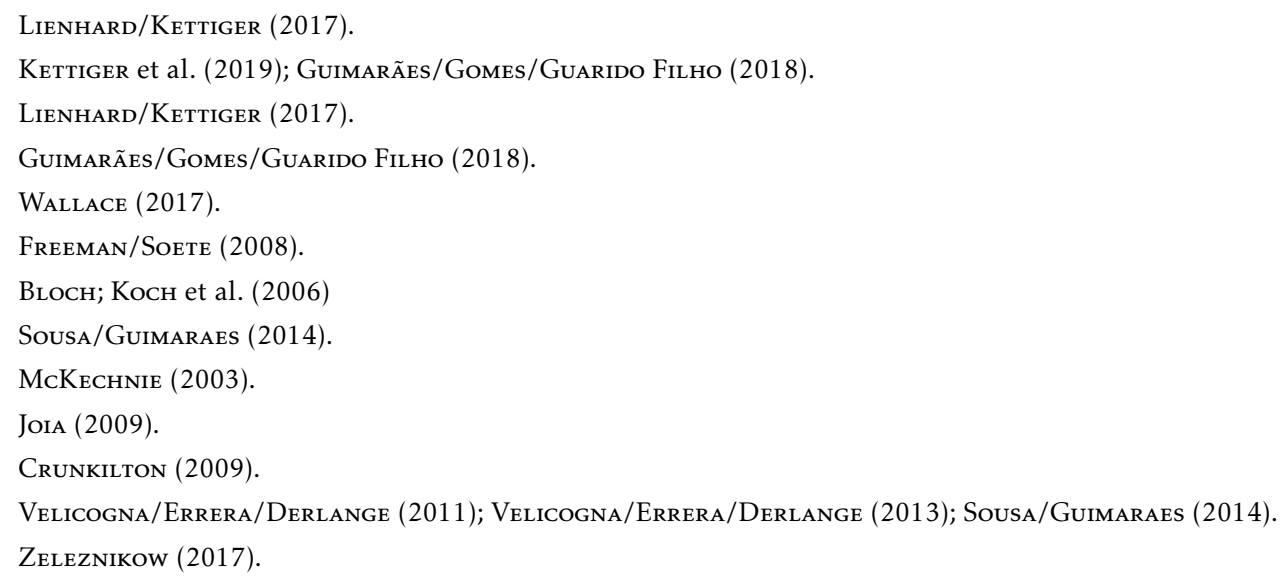


and parties/lawyers through a platform (Justitia.Swiss).» ${ }^{14}$ Im neuesten Bericht der European Commission for the efficiency of justice (CEPEJ) liegen keine Informationen über den Prozentsatz der in elektronischen Verfahren bearbeiteten Fälle vor. Es wird die Frage nach der Möglichkeit zur elektronischen Einreichung eines Falles an die Gerichte gestellt, sodass im Jahr 2016 die entsprechende Einsatzrate in der Schweiz in Zivilverfahren 50-99\%, in Strafverfahren 50-99\% und in Verwaltungsverfahren $10-49 \%$ betrug. ${ }^{15}$ Die Studie ist im Schweizer Kontext auf das Projekt Justitia 4.0 ausgerichtet.

\subsection{Fokus der Studie}

[5] Vor dem Hintergrund, dass es noch wenige empirische und theoretische Studien im Zusammenhang mit der Schweizer Justiz gibt, ${ }^{16}$ versucht diese Studie die folgende Frage zu beantworten: Was sind die wichtigsten Schlüsselfaktoren, d.h. Triebkräfte und Hindernisse, bei der Digitalisierung von Gerichten in der Schweiz? Für die vorliegende Publikation liegt der Schwerpunkt auf Innovationen im technologischen Bereich, genauer gesagt auf den Informations- und Kommunikationstechnologien, die von den Gerichten entwickelt und eingesetzt werden. Ziel der Publikation ist es, die Erfahrungen und Strategien zur Einführung von Innovationen zu beschreiben, die relevanten Schlüsselfaktoren der Einführung von Innovationen zu diskutieren und die erwarteten Ergebnisse und Auswirkungen der Einführung von E-Gerichten zu identifizieren. Die hier vorgestellten Ergebnisse beziehen sich auf ein transnationales Projekt, an dem Gerichte in der Schweiz und in Brasilien beteiligt sind. Dieser Beitrag stellt die Untersuchung bezüglich der Gerichte in der Schweiz dar.

\section{Hintergrund: Innovation im öffentlichen Sektor}

[6] Im öffentlichen Sektor sind die Entwicklung, Einführung und Evaluierung von Innovationen komplex, haben jedoch in den letzten Jahren durch die Schaffung von Forschungsprogrammen und Auszeichnungen an Aufmerksamkeit gewonnen. In Europa können wir den European Public Sector Award (EPSA), PUBLIN, das öffentliche Innovationsforschungsprogramm der Europäischen Union und den MEPIN (Measuring innovation in the public sector in the Nordic countries) erwähnen. Innerhalb der europäischen Justiz gibt es den Preis Kristallene Waage der Justiz, «aimed at discovering and highlighting innovative and efficient practices concerning the functioning of justice, judicial procedures or the organisation of courts.» ${ }^{17}$

[7] Innovation «is an idea, practice, or object that is perceived as new by an individual or other unit of adoption.» ${ }^{18}$ Die Verbreitung von Innovationen wird verstanden als «acceptance over time, of some specific item - an idea or practice, by individuals, groups or other adopting units, linked to specific channels of communication, to a social structure, and to a given system of

\footnotetext{
BÜHLER (2018), 25.

CEPEJ.

Lienhard/Kettiger (2017).

CEPEJ.

Rogers (2003), 11.
} 
values, or culture.» ${ }^{19}$ Der Entscheidungsfindungsprozess für die Umsetzung und Verbreitung von Innovationen umfasst fünf Schritte: (i) Wissen - Entdecken und Verstehen der Innovation; (ii) Überzeugungskraft - Bildung einer positiven oder negativen Haltung gegenüber der Innovation; (iii) Entscheidung - Entscheidung über Umsetzung oder Ablehnung; (iv) Umsetzung effektive Nutzung der Innovation; und (v) Bestätigung - Festigung der Umsetzung in deren Anwendung. ${ }^{20}$

[8] Rogers ${ }^{21}$ hat fünf Merkmale aufgezeigt, welche die Umsetzung und die Umsetzungsrate einer Innovation beeinflussen: (i) relativer Vorteil - die Umsetzung ist in dem Masse vorteilhaft, in dem die Innovation offensichtliche Vorteile in Bezug auf das Produkt, die Dienstleistung oder das aktuelle Verhalten bietet; (ii) Kompatibilität mit Systemen und Werten - je mehr die Innovation mit der vorher bestehenden Situation kompatibel ist, desto grösser sind die Chancen und ist die Rate ihrer Umsetzung; (iii) Komplexität - Einfachheit der Umstellung - je komplexer die mit der Innovation verbundenen Veränderungen sind, desto geringer sind die Chancen und ist die Geschwindigkeit ihrer Umsetzung; (iv) Möglichkeit des Testens - das Testen einer Innovation, bevor eine endgültige Entscheidung getroffen wird, erhöht die Chancen und die Geschwindigkeit ihrer Umsetzung; und (v) Sichtbarkeit der Veränderung und ihres Nutzens - je offensichtlicher die Vorteile einer Innovation sind, desto wahrscheinlicher und schneller wird sie umgesetzt. Diese Merkmale werden als die wichtigsten Schlüsselfaktoren in Innovationsstudien im öffentlichen Sektor genannt. ${ }^{22}$ Eine Innovation ist nicht zwangsläufig erwünscht ${ }^{23}$ oder führt nur zu den erwarteten Ergebnissen. Auch kann es immer noch zu einer Ablehnung der Innovation kommen.

[9] Innovation ist ein relevantes Thema im Forschungsbereich der Justizverwaltung, ${ }^{24}$ und im technologischen Bereich ist die Entwicklung und Umsetzung von ICTs in den Gerichten hervorzuheben. ${ }^{25}$ Doch nur wenige Länder haben tatsächlich Erfolg mit integrierten und automatisierten Systemen für Gerichtsverfahren. ${ }^{26}$ Ursprünglich wurden die ICTs nicht als Mittel zur Verbesserung der internen Verwaltung der Gerichte angesehen, sondern mit viel Kritik konfrontiert. ${ }^{27}$

[10] Gerichte und Richter standen in den letzten Jahren unter dem Druck, bessere Leistungen zu erbringen und so hat die Leistungsfähigkeit der Justiz an Bedeutung gewonnen. ${ }^{28}$ Im Einklang damit wurden Vereinfachungs- und Digitalisierungsstrategien von mehreren Ländern bei Justizreformen eingesetzt, um Engpässe bei der Leistungsfähigkeit der Gerichte zu beseitigen. ${ }^{29}$ Online-Zugang zur Justiz, insbesondere in weniger komplexen Fällen, trägt zu Kosten- und Zeiteinsparungen bei, da Fälle schneller, leichter und besser gelöst werden. ${ }^{30}$ Auf der einen Seite besteht ein direkter und positiver Zusammenhang zwischen Investitionen in ICT und der Produkti-

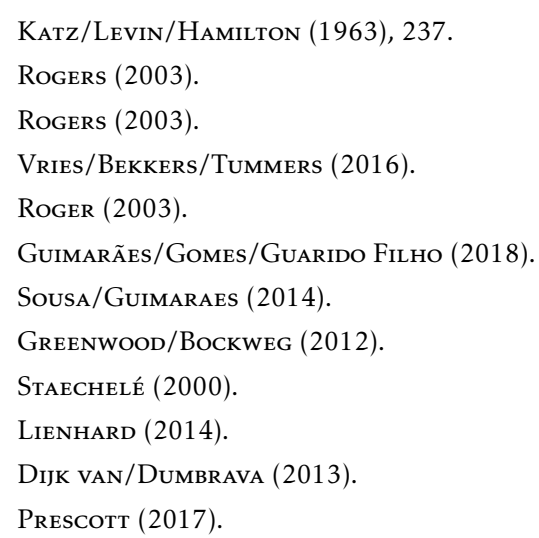


vität der Gerichte, ${ }^{31}$ wie für die Staats- und Bundesgerichte in Brasilien festgestellt wurde. Andererseits, was die Schnelligkeit und Dauer eines Gerichtsverfahrens betrifft, reicht der Einsatz von ICTs allein nicht aus, um die Dauer eines Gerichtsverfahrens zu verkürzen. ${ }^{32}$ Wie die Erkenntnisse zeigten, ist der Vergleich zwischen der Dauer von physischen und elektronischen Steuervollstreckungsfällen im brasilianischen Kontext statistisch nicht signifikant. PROCoPIUCK weist auch darauf hin, dass die Gesetzgebung, neben dem Management und der Technik, ebenfalls ein wichtiger Faktor darstellt, der sich auf die Leistungsfähigkeit der Justiz auswirkt. ${ }^{33}$

[11] Selbstverständlich sind Geschwindigkeit und Produktivität nicht die einzigen Massstäbe für Leistung und Effektivität beim Einsatz von ICTs an Gerichten. Wie Artikel 6 der Europäischen Menschenrechtskonvention festhält, hat jeder das Recht auf ein «[...] fair and public hearing within a reasonable time by an independent and impartial tribunal established by law.» ${ }^{34}$ Juristische Qualität umfasst auch technologische Tools und Datenbanken. ${ }^{35}$ Durch den verstärkten Einsatz von ICTs können die Gerichte den Zugriff, die Transparenz, die Fairness und die Gleichberechtigung verbessern, sind aber auch den Risiken eines entmenschlichten Justizsystems ausgesetzt. ${ }^{36}$

[12] Zahlreiche Technologien haben dazu beigetragen, die am Justizprozess beteiligten Parteien besser zu unterstützen, wodurch die Effektivität und Legitimität der Justiz verbessert werden konnten. In diesem Zusammenhang wird hervorgehoben, wie wichtig es ist, die Bedürfnisse externer Nutzer bei alltäglichen Technologien zu erfüllen, z.B. bei der Nutzung von Mobiltelefonen zur Beschaffung und Versendung von Informationen und Dateien, auch als Beweismittel vor Gericht, bei Videokonferenzen unter Einbeziehung der betroffenen Parteien und des Anwalts, bei der Planung von Anhörungen nach Wunsch der Parteien, bei der Online-Streitschlichtung ${ }^{37}$ usw.

[13] Die Literatur hat gezeigt, wie wichtig die Anwendung neuer ICTs im Justizwesen ist: Videokonferenzen bei Fernanhörungen vor französischen Gerichten; ${ }^{38}$ Online-Streitschlichtung in Brasilien; ${ }^{39}$ Annäherung der Gerichte an die Bevölkerung durch den Einsatz von Live-Web-Streaming und sozialen Netzwerken wie Blogs, Twitter und Facebook, ${ }^{40}$ YouTube, Linkedln. ${ }^{41}$ Diese neuen Technologien können dazu beitragen, das Vertrauen in die Justiz zu stärken. ${ }^{42}$

[14] Insbesondere in Bezug auf E-Gerichtssysteme sind vor kurzem Studien zu diesem Thema mit einer Vielzahl von Fragestellungen und Kontexten veröffentlicht worden: Umsetzung und Implementierung des elektronischen Gerichtsverfahrens im Staatsgericht des Bundesbezirks Brasilia, aus der Sicht der Anwälte; ${ }^{43}$ Entwicklung und Umsetzung des elektronischen Gerichtsverfahrens in den Bundesarbeitsgerichten in Brasilien aus der Sicht der Richter und Gerichtsmanager; ${ }^{44}$

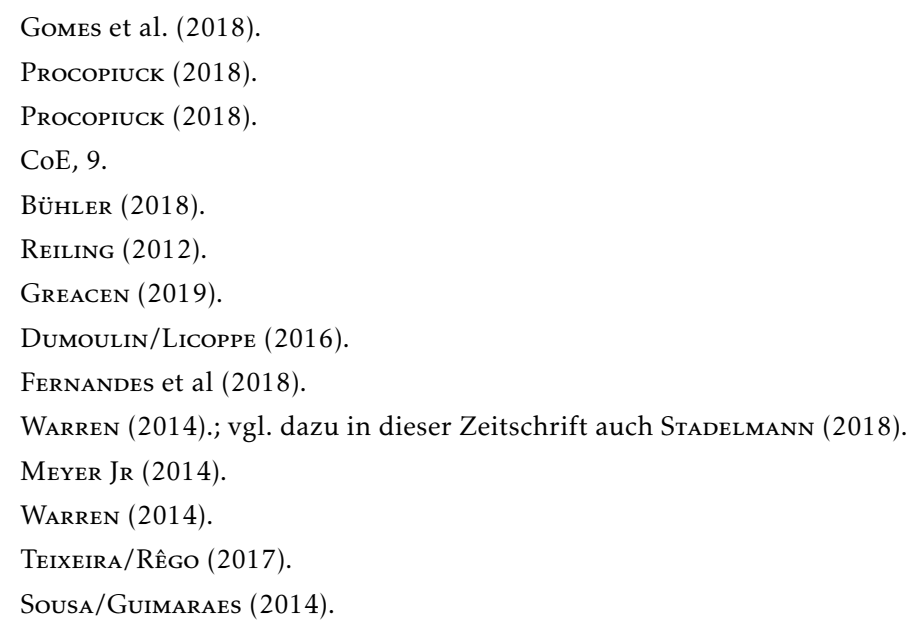


Schlüsselfaktoren und Ergebnisse der Umsetzung des elektronischen Verarbeitungssystems am brasilianischen Obersten Gerichtshof; ${ }^{45}$ Darstellung des Systems zur elektronischen Beweismittelverwaltung beim Internationalen Strafgerichtshof in Den Haag, Niederlande; ${ }^{46}$ elektronische Fallanalyse und -beilegung in Berufungsgerichten; ${ }^{47}$ Umsetzung des E-Codex - Projekt für Europäische Zahlungsaufträge in europäischen Ländern; ${ }^{48}$ Besprechung von Risikofaktoren im Zusammenhang mit dem Entwurf, der Entwicklung und der Umsetzung von E-Justiz-Systemen in Singapur, Brasilien, Belgien, Portugal und Kap Verde; ${ }^{49}$ Diskussionen über die e-Discovery in nordamerikanischen Staats- und Bundesgerichten; ${ }^{50}$ Umsetzung einer elektronischen Fallbearbeitung an zwei Gerichten und in zwei Staatsanwaltschaften in Italien. ${ }^{51}$

[15] Somit steht diese Publikation im Kontext von Studien über die Umsetzung von ICTs und E-Government, genauer gesagt mit E-Gerichtsstrategien in der Justiz. Der nächste Abschnitt befasst sich mit den methodologischen Aspekten, die zur Ermittlung der Ergebnisse verwendet wurden.

\section{Methodologie}

[16] Der Ansatz der Forschung war deskriptiv und qualitativ. Gegenstand sind die von den Schweizer Gerichten entwickelten und umgesetzten technologischen Innovationen. In der Schweiz wurde die Umsetzung des Projekts Justitia 4.0 evaluiert. Dies erfordert vorab die Auseinandersetzung mit Justitia 4.0, da es sich um ein Projekt handelt, das sich in der Umsetzung befindet und alle Gerichtsbarkeiten der Schweiz umfassen wird.

[17] Um eine gewisse Repräsentativität hinsichtlich der Bevölkerung zu erhalten, wurden sieben Kantone ausgewählt, mit Priorität für die ersten Kantone, die den öffentlich-rechtlichen Zusammenarbeitsvertrag mit der Schweizerischen Eidgenossenschaft unterzeichnet hatten, vertreten durch das Schweizerische Bundesgericht und die am Projekt beteiligten Personen. Tabelle 1 zeigt die Kantone und Gerichte der ersten und zweiten Instanz, die in der Schweiz untersucht wurden. Es wurden insgesamt 22 Interviews mit neun Richtern, fünf Informatikverantwortlichen und acht Justizmanagern durchgeführt. Eine der Befragten war eine Frau und 21 der Befragten waren Männer. Das Durchschnittsalter der Befragten betrug 51 Jahre, mit einer Standardabweichung von 9,5 Jahren. Sie verfügten über eine durchschnittliche Gerichtspraxis von etwa 15 Jahren, mit einer Standardabweichung von etwa 12 Jahren.

45 Freitas/Medeiros (2015).

46 Dillon/BERESFORd (2014).

47 Magnuson/Thumma (2014).

48 Pangalos/Salmatzidis/Pagkalos (2014).

49 Rosa/Teixeira/Pinto (2013).

50 CARLson (2007).

51 Contini (2000). 
Tabelle 1: Kantone und Gerichte in der Schweizer Forschung

\begin{tabular}{cl}
\hline Kanton & \multicolumn{1}{c}{ Gericht } \\
\hline Zürich & Oberstes Gericht des Kantons Zürich \\
\hline Justizverwaltung des Kantons Bern & Verwaltungsgericht des Kantons Bern \\
Luzern & Kantonsgericht des Kantons Luzern \\
Thurgau & Regionales Zivilgericht des Kantons Luzern \\
Neuchâtel & Oberstes Gericht des Kantons Thurgau \\
Basel-Landschaft & Kantonsgericht des Kantons Neuenburg \\
Waadt & Kantonsgericht des Kantons Basel-Landschaft \\
& Kantonsgericht des Kantons Waadt \\
& Bezirksgericht Lausanne \\
\hline Bundesebene & Schweizerisches Bundesgericht \\
\hline
\end{tabular}

Quelle:52 Forschungsdaten.

[18] Die Daten wurden im Zeitraum von 2019 bis 2020 in zwei Phasen erhoben. Die erste bestand aus einer bibliographischen Studie, um den theoretischen Hintergrund und den Stand der Technik des Themenbereichs zu ermitteln. In der zweiten Phase wurden die erwähnten teilstrukturierten ausführlichen Interviews geführt.

[19] Die Interview-Scripts, auf welche Bezug genommen wird, ${ }^{53}$ wurden so strukturiert, dass folgende Aspekte berücksichtigt wurden: die Eigenheiten von Gerichtsinnovationen, die Dynamik der Planung und der Umsetzung von Innovationen, die Schlüsselfaktoren, d.h. Aspekte, die zur Umsetzung von Innovationen beigetragen und diese erschwert haben, und die Einschätzung der erwarteten Ergebnisse und Auswirkungen.

[20] Die durch die Interviews gesammelten Informationen wurden aufgezeichnet, transkribiert, zur Bestätigung und Validierung der Daten an die Befragten zurückgesandt und mit der von

52 Bundesgericht.

53 Sousa and Guimaraes (2017). 
BARDIN vorgeschlagenen Inhaltsanalysentechnik ${ }^{54}$ in drei Schritten analysiert: (i) Voranalyse, Sondieren der Literatur, Aufbau eines Corpus; (ii) Auswertung des Materials durch den Aufbau einer Datenbank, wobei Auszüge aus den Interviews mit den definierten Kategorien und den Variablen verknüpft werden, und (iii) Behandlung und Interpretation der Ergebnisse gemäss den Zielsetzungen und Kategorien der Analyse.

[21] Die Analysekategorien wurden definiert als: Schlüsselfaktoren für Innovation, d.h. Aspekte, die zur Umsetzung der Innovationen beitragen, und Aspekte, die die Umsetzung der untersuchten Innovationen erschweren; Ergebnisse und Auswirkungen. Die Schlüsselfaktoren wurden, wie von VRIEs et. al. vorgeschlagen, in Untersuchungsebenen eingeteilt ${ }^{55}$ : in die Kategorien Umfeld, Organisation, Innovation und Individuum. Die Kategorien wurden in Tabellen mit den Ergebnissen nach Interviewgruppen und Häufigkeit der Antworten dargestellt.

\section{Ergebnisse und Diskussion}

[22] Im Jahr 2016 wurden 68,6\% des Schweizer Justizbudgets für die Computerisierung der Gerichte bereitgestellt. ${ }^{56}$ In der Schweiz ist das Projekt «Justitia 4.0», das unter anderem das funktionale Ziel hat, das "One-Stop-Shop»-Portal der Schweizer Justiz zu werden, ${ }^{57}$ in der Konzeptionsphase und soll bis 2026 vollständig implementiert sein. Um das Projekt zu realisieren, wird zudem ein Gesetzgebungsprojekt, enthaltend eine Überprüfung und Anpassung der schweizerischen Zivil- und Strafprozessordnung, durchgeführt. ${ }^{58}$

[23] Der Name des Projekts ist von dem Fachbegriff Industrie 4.0 abgeleitet, wobei dieser Begriff mit einer Phase der industriellen Revolution verbunden ist und 2011 auf der Messe in Hannover von der deutschen Regierung vorgestellt wurde. ${ }^{59}$ Dem Begriff der Industrie 4.0 wurden folgende Komponenten der technologischen Revolution zugeschrieben: «big data, autonomous robots, augmented reality, additive manufacturing, cloud computing, cyber security, internet of things, system integration and simulation.» ${ }^{60}$ Insofern ist es durchaus gewagt, ein Projekt im Justizkontext so zu nennen, insbesondere dann, wenn es primär um den Aufbau einer Austauschplattform geht.

[24] Tabelle 2 zeigt die wichtigsten Schlüsselfaktoren von Innovationen, gegliedert nach der Untersuchungsebene und nach den befragten Personen. Je mehr «+» diese Schlüsselfaktoren erhielt, desto häufiger wurde sie von den Befragten erwähnt.

\footnotetext{
54 BARDin (2011).

55 VRIEs et al. (2016).

56 CEPEJ.

57 HIS-Programm.

58 BüHLER (2018).

59 Bal/Erkan (2019).

60 BAL/ERKAN (2019), 626.
} 
Tabelle 2: Schlüsselfaktoren für Innovation (Schweizer Teil des Forschungsprojekts)

\begin{tabular}{|c|c|c|c|c|c|}
\hline Ebene & Triebkräfte / Hindernisse & $\begin{array}{l}\text { Richter } \\
(\mathrm{N}=9)\end{array}$ & $\begin{array}{l}\text { IT Manager } \\
(\mathrm{N}=5)\end{array}$ & $\begin{array}{l}\text { Justiz Manager } \\
(\mathrm{N}=8)\end{array}$ & $\begin{array}{l}\text { Summe } \\
(\mathrm{N}=22)\end{array}$ \\
\hline \multirow{7}{*}{$\begin{array}{l}\frac{\bar{\sigma}}{\omega} \\
\stackrel{\underline{\xi}}{g}\end{array}$} & Zugang & +++ & ++ & ++++ & ++++ \\
\hline & Gesetzgebung & ++ & +++ & +++ & +++ \\
\hline & Föderalismus & + & +++ & ++++ & +++ \\
\hline & Unabhängigkeit & + & / & ++ & + \\
\hline & Andere Sprachen & / & + & / & + \\
\hline & Integration mit anderen & & & & \\
\hline & e-gov-Systemen & + & I & + & + \\
\hline \multirow{16}{*}{ 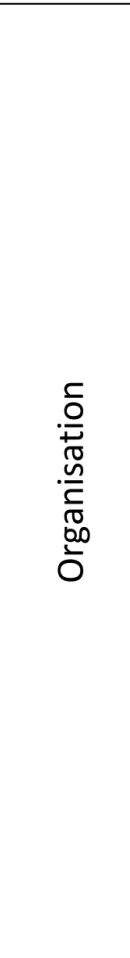 } & $\begin{array}{l}\text { Langfristige Umsetzung } \\
\text { Aktualisierung und } \\
\text { Anpassung früherer }\end{array}$ & ++ & + & ++++ & +++ \\
\hline & Systeme & ++ & ++ & +++ & ++ \\
\hline & Kommunikation / & & & & \\
\hline & Weiterbildung & + & + & ++++ & ++ \\
\hline & $\begin{array}{l}\text { Konkrete Ergebnisse } \\
\text { Klarer Fokus und }\end{array}$ & ++ & / & +++ & ++ \\
\hline & Zielsetzung & / & +++ & +++ & ++ \\
\hline & Kooperation / Mitarbeit & + & + & ++ & + \\
\hline & Unterstützung & ++ & + & / & + \\
\hline & $\begin{array}{l}\text { Interdisziplinäres Team } \\
\text { Zahl der beteiligten }\end{array}$ & + & + & + & + \\
\hline & Personen & / & +++ & / & + \\
\hline & Einsatz von & & & & \\
\hline & Hybridsystemen & + & I & + & + \\
\hline & Leitung & / & / & ++ & + \\
\hline & Überschneidung der & & & & \\
\hline & Projekte & / & + & / & + \\
\hline & Engagierte Mitarbeiter & 1 & + & I & + \\
\hline \multirow{3}{*}{ 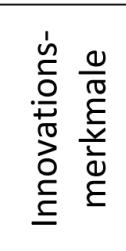 } & Anwendbarkeit & ++ & + & ++++ & +++ \\
\hline & (Isolierte Testumgebung) & + & + & I & + \\
\hline & Sicherheit / Datenschutz & l & ++ & +++ & ++ \\
\hline \multirow{4}{*}{$\begin{array}{l}\frac{\xi}{5} \\
\frac{2}{2} \\
\frac{0}{2} \\
\frac{0}{0} \\
\underline{\underline{0}}\end{array}$} & Widerstand & ++++ & + & +++ & +++ \\
\hline & Alter & +++ & ++++ & / & ++ \\
\hline & $\begin{array}{l}\text { Vorhergehende Erfahrung } \\
\text { im Umgang mit e-gov und } \\
\text { anderen elektronischen }\end{array}$ & & & & \\
\hline & Tools. & / & +++ & + & + \\
\hline
\end{tabular}

Quelle: Forschungsdaten. 
Anmerkungen: ++++ steht für einen Schlüsselfaktor, der von mindestens zwei Dritteln der Befragten genannt wird; +++ von mindestens der Hälfte der Befragten erwähnt; ++ von mindestens einem Viertel der Befragten erwähnt; + mindestens einmal erwähnt; / Schlüsselfaktor nicht erwähnt.

[25] Zugang war nach Meinung der Befragten der wichtigste Schlüsselfaktor auf der Umfeldebene. Zugang wurde in zweifacher Hinsicht interpretiert, nämlich als erleichterter Zugang zu Informationen für die an einem Gerichtsverfahren beteiligten Parteien und als Zugang zur Justiz für die Bevölkerung. Wie ein Befragter erwähnt, «wird das Informationssystem es Ihnen ermöglichen, überall auf Informationen zuzugreifen, Informationen zu suchen und zu vergleichen, sich auf die wichtigsten Informationen zu konzentrieren und sie unmittelbar mit anderen Parteien zu teilen.» Ferner aus einer umfassenderen Perspektive, "denke ich, dass es den Zugang der Bevölkerung zu den Gerichten erleichtern wird, und dies ist ein sehr wichtiges Thema auf demokratischer Ebene, denn der Zugang zu den Gerichten ist für die Funktionsfähigkeit der Demokratie von grundlegender Bedeutung.» Diese Voraussetzungen wurden auch von Freitas und Medeiros $^{61}$ im brasilianischen Obersten Gerichtshof, in der Studie von TeIXeIra und RêGo ${ }^{62}$ im Staatsgericht des Bundesbezirks in Brasilien und in den US-Bundesgerichten erkannt. ${ }^{63}$

[26] Die Gesetzgebung war ein wichtiger Aspekt, der von allen Befragten in zweierlei Hinsicht hervorgehoben wurde. Erstens die neue Gesetzgebung auf Bundesebene, die die elektronische Kommunikation zwingend vorschreibt. Wie ein Befragter sagt: «Wir brauchen neue Gesetze, wenn wir den elektronischen Weg einschlagen, dann muss auch alles digitalisiert werden, dann müssen Papierakten beiseitegelegt werden, also brauchen wir Gesetze, die das zwingend vorschreiben.» Zweitens eine Gesetzgebung auf kantonaler Ebene, die das für die Umsetzung erforderliche Budget festlegt. Im Allgemeinen muss eine ICT-basierte Innovation bei den Gerichten auch Rechtsvorschriften und Verwaltungsverfahren vorsehen, die implementiert werden. ${ }^{64} \mathrm{Ob}-$ wohl in einer früheren Studie eine obligatorische Umsetzung als Hindernis betrachtet wurde, ${ }^{65}$ waren die Schweizer Teilnehmenden der Meinung, dass im Falle der Schweiz eine solche Gesetzgebung eine wichtige Triebkraft darstellen würde.

[27] Oft erwähnt wurde der Föderalismus. Dies ist ein spezifischer Aspekt im schweizerischen Kontext, den zwei der Manager erwähnen: «Der Föderalismus ist hinderlich, wir haben 26 Kantone und zahlreiche Meinungsträger, und da ist es nicht einfach, zu jedem dieser Aspekte das gleiche Verständnis, die gleichen Ziele, die gleichen Meinungen zu haben» und «Das Problem in der Schweiz ist, dass es 26 verschiedene Kantone mit ihrem eigenen Justizsystem gibt.» Der mit der kantonalen Autonomie und einem demokratischen Charakter verbundene Föderalismus des Landes bringt Komplexität in die Entwicklung und Umsetzung von Innovationen im grossen Stil, wie zwei Manager erläutern: «Ehrgeizige Projekte, sehr komplex wegen der Vielzahl von Interessengruppen [...] in der Schweiz»; «Wir versuchen, einen Konsens zu erreichen, wir suchen danach, um Entscheidungen zu treffen, wenn wir keinen konkreten Konsens erreichen können, wird es schwierig.» In derselben länderspezifischen Rubrik wurde nur von IT-Verantwortlichen erwähnt, dass Sprache, wo doch die Schweiz vier Amtssprachen hat, ein Hindernis sein kann.

61 Freitas/Medeiros (2015),

62 TeIXeIra/RÊGo (2017).

63 Greenwood/Bockweg (2012).

64 WEERs (2016).

65 Sousa/Guimaraes (2014). 
[28] Die Unabhängigkeit der Justiz wurde von den Richtern und Justizmanagern als Schlüsselfaktor angesehen, dies auch mit Bezug auf Artikel 191c der Bundesverfassung der Schweizerischen Eidgenossenschaft, der besagt, dass die Justizbehörden in der Ausübung ihrer richterlichen Tätigkeiten unabhängig und allein dem Gesetz unterworfen sind. Die Befragten äusserten sich besorgt über mögliche Ausuferungen der Transparenz und über erhöhten Druck durch Statistiken und Leistungsmessungen.

[29] Schliesslich wurde auf Umfeld-spezifischer Ebene auch die Notwendigkeit einer Integration mit anderen staatlichen Systemen hervorgehoben. Die Systemintegration kann viel zur Verbesserung der Dienstleistungen beitragen. In Kanada ist zum Beispiel das Case Management System der Strafverfolgungsbehörden zur Erfassung von Daten der Polizei und anderer staatlicher Instanzen integriert. ${ }^{66}$ Die Kompatibilität mit anderen Regierungssystemen wurde auch in anderen Studien als ein wichtiger Schlüsselfaktor angesehen. ${ }^{67}$

[30] Die Untersuchungsebene mit der grössten Anzahl von Schlüsselfaktoren war die organisatorische Ebene. Der zeitliche Rahmen war ein Punkt, der auf dieser Ebene angesprochen wurde, jedoch mangelte es in diesem Aspekt an Einigkeit. Einige sind überzeugt, es handle sich um einen sehr langen Zeitrahmen bei der Entwicklung und dem Einsatz: «[...] wir haben 2018 begonnen, und wir sind gespannt auf 2026, wobei ich mir nicht allzu sicher bin, ob wir es bis 2026 schaffen werden, vielleicht erst 2030, sehen Sie, das ist eine lange Zeit. Und wenn Sie über technische Innovationen in diesem Zeitraum nachdenken, dann ist die Technik im Jahr 2030 vielleicht ganz anders, als wir sie heute haben.» Andere sind überzeugt, dass dies für eine reibungslose Implementierung der neuen Technologie und eine sorgfältige Planung der Umstellung wichtig sein wird, um Risiken zu vermeiden: «Es ist lang, aber ich bin mir sicher, dass wir eine gute Lösung finden werden, das dauert lange»; «Es wird eine lange Phase geben, in der sich die Menschen anpassen müssen und in der sich die Abläufe innerhalb der Gerichte anpassen müssen.» Und: «Ich empfehle keinen «Big Bang`-Ansatz, weil das Risiko des Scheiterns hoch ist. Eine schrittweise Einführung, die es den Menschen ermöglicht, ihre Arbeitsabläufe nach und nach umzustellen, ist angebracht.»

[31] Ein Anliegen, das von allen Gruppen der Befragten hervorgehoben wurde, waren die gegenwärtig von den Gerichten verwendeten Systeme. Ihnen ist bewusst, dass sie die derzeit verwendeten Systeme aktualisieren und an das Projekt Justitia 4.0 anpassen müssen, wie ein Manager erwähnte: «Wir sind mit [dem derzeitigen System] zufrieden, es ist heute perfekt, aber [es] muss auch in Zukunft optimiert werden, und wir sind uns gegenwärtig nicht sicher, ob sie in der Lage sind, die Software zu verbessern, dies ist heute eines unserer Hauptanliegen.» Im Zusammenhang mit diesem Schlüsselfaktor wurde auch die Verwendung von Hybridsystemen erwähnt, d.h. dass zunächst zwei Systeme verwendet werden müssen, um die gleichen Daten freizugeben, oder dass man immer noch gleichzeitig in einem elektronischen und einem physischen System arbeiten muss. Frühere Studien deuten darauf hin, dass sich dies negativ auf die Leistungsfähigkeit der Justiz auswirken und zu Mehrarbeiten führen kann. ${ }^{68}$

[32] Angesichts der Komplexität und des Ausmasses des Projekts unterstrichen die Befragten die Bedeutung der Kommunikation in allen Phasen, bei den Zielen und dem Einsatz der Innovation

66 BORKOWSKI (2004).

67 Freitas/Medeiros (2015); Sousa/Guimaraes (2014).

68 Sousa/Guimaraes (2014); Lienhard/Kettiger/Uster/Winkler (2015). 
sowie bei den organisatorischen Weiterbildungsmassnahmen. Gut strukturierte Schulungen und Implementierungshandbücher werden als wesentlich für die Umsetzung einer Innovation in der Gerichtspraxis angesehen. ${ }^{69}$ In dieser organisatorischen Betrachtung wurde auch die Bedeutung der Beteiligung und Kooperation der Betroffenen und den interdisziplinären Entwicklungsteams erwähnt. Das Projekt Justitia 4.0 hat Expertengruppen mit Vertretern aus verschiedenen Bereichen und von verschiedenen Gerichten gebildet. Diese Strategie findet sich auch in bisherigen Studien zur Innovation. ${ }^{70}$

[33] Insbesondere im Zusammenhang mit der Implementierung des Projekts vertraten die Befragten die Ansicht, dass einige Elemente dazu beitragen könnten, Hindernisse für eine Umsetzung abzubauen: (i) die Betroffenen mit konkreten Resultaten bezüglich der Nutzung der Innovation zu konfrontieren; (ii) klare Schwerpunkte und Ziele zu setzen; (iii) Unterstützung bei der Implementierung und Integration in bestehende Informationssysteme; (iv) auch in der Literatur wurde Führungskompetenz als ein wichtiger Schlüsselfaktor in allen Phasen des Innovationsprozesses angesehen $;^{71}(\mathrm{v})$ insbesondere IT-Manager glauben, dass einerseits viele Personen am Projekt beteiligt sind und andererseits nur eine sehr begrenzte Anzahl von Mitarbeitenden in Vollzeit am Projekt beteiligt ist, und (vi) ausserdem denken nur IT-Manager, dass es bei einigen Teilprojekten Überschneidungen geben kann.

[34] Es gab drei Apekte von Innovationsmerkmalen: Anwendbarkeit, Sandboxes und Datenschutz-/Sicherheitsfragen. Die Anwendbarkeit wurde mit Benutzerfreundlichkeit assoziiert, wie zwei Befragte erwähnten. Einer betonte den Nutzen einer einfachen Bedienung: «Wichtig ist, dass wir ein einfaches System haben, das nicht zu kompliziert für die Mitarbeitenden ist.» Und der andere betonte die Wahrnehmung der Nützlichkeit: «[...] es wird die Arbeitsabläufe verändern, und es wird einfacher sein, denke ich, denn Papier ist nicht sehr benutzerfreundlich, wenn man etwas recherchiert.» Diese Benutzerfreundlichkeit bzw. die gefühlte Nutzbarkeit wurde auch in der Literatur als relevanter Schlüsselfaktor genannt. ${ }^{72}$ Interessant ist zu bemerken, dass die Anwendbarkeit von IT-Managern weniger erwähnt wurde.

[35] Die Möglichkeit, eine Innovation auszuprobieren oder zu testen, bevor eine endgültige Entscheidung getroffen wird, erhöht die Wahrscheinlichkeit, dass sie umgesetzt wird. ${ }^{73}$ Im gleichen Sinne wurden Sandboxes als ein wichtiger Schlüsselfaktor wahrgenommen. In der Einschätzung eines Managers: «Soweit ich weiss, was ich für sehr gut halte, werden sie diese Lösung zunächst anhand von Sandboxes realisieren, im Rahmen von kleineren Projekten, um alle produktiven oder vorproduktiven Gesetzgebungen in Pilotprojekten auszuprobieren und sie dann sukzessiv in den Kantonen umzusetzen.» Die Sandboxes sind Teil der Methodik des Projekts Justitia 4.0. «Sandboxes» sind kleinere produktive oder produktionsnahe Piloten in einzelnen Kantonen respektive einzelnen Gerichten oder Staatsanwaltschaften, mit denen die Benutzerfreundlichkeit, die Gesetzeskonformität, die technische Machbarkeit und die administrativen Prozesse der zukünftigen Anwendungen schon zu einem frühen Zeitpunkt getestet werden können. Die An-

69 Sousa/Guimaraes (2014); Greenwood/Bockweg (2012).

70 Sousa/Guimaraes (2014); Greenwood/Bockweg (2012).

71 Castro/Guimaraes (2014); Baxter/Schoeman/Goffin (2011).

72 Davis (1993); Davis/Bagozzi/Warshaw (1989); Sousa/Guimaraes (2014).

73 Rogers (2003). 
wendungen haben zu diesem Zeitpunkt eingeschränkte Funktionalitäten. «Je nach Fragestellung kann sich eine 〈Sandbox〉 auch auf eine Konzeptstudie oder einen Prototypen beschränken». ${ }^{74}$

[36] Justizmanager und IT-Manager haben auf die Relevanz des Datenschutzes und der Datensicherheit hingewiesen, wobei zwei Manager feststellen, dass «in der Schweiz die Sicherheit einen höheren Stellenwert hat als der Zugang.» Und: «Die Menschen wollen ihre Daten schützen, [...] wir brauchen Sicherheit, wir brauchen Vertrauen [in das System].» Das Potenzial für Sicherheitsverletzungen stellt ein starkes Hindernis für die Umsetzung von ICTs in Gerichten dar. ${ }^{75}$ Sicherheit, Integrität und Vertraulichkeit von Daten und Informationen sind in dieser Hinsicht wesentliche Elemente. ${ }^{76}$

[37] Auf der individuellen Ebene wurden drei Schlüsselfaktoren identifiziert: Widerstand, Alter und vorhergehende Erfahrung bei der Nutzung von Online-Diensten. Der Widerstand gegenüber Veränderungen ist ein wichtiger Schlüsselfaktor, der ein Hindernis für Innovationen darstellt. Aber die Notwendigkeit zur Veränderung ist für einige zwingend notwendig, wie ein Befragter erwähnt: «Wir stehen noch ganz am Anfang, aber bisher ist es sehr schön zu sehen, dass es meiner Meinung nach in der ganzen Schweiz wirklich viele Menschen gibt, die denken, es müsse etwas getan werden. Wir sind noch etwas im Rückstand. Wir müssen vorankommen, denn wir sind mit dem E-Government im Rückstand, und deshalb ist es wirklich wichtig, in diesen Digitalisierungsprozess bei den Gerichten einzusteigen. Es ist daher sehr gut zu sehen, dass die Menschen wirklich vorankommen wollen.» Dieser Schlüsselfaktor findet sich auch in der Literatur als Hindernis. $^{77}$

[38] Das Alter wurde von den Befragten, in der gleichen Weise wie bereits in der Literatur zur Gerichtsverwaltung beschrieben, als Generationsunterschied wahrgenommen. In dem Sinne, dass ältere Berufstätige mehr Widerstand bei der Umsetzung von Innovationen und mehr Schwierigkeiten beim Zugriff und der Nutzung dieser Nachrichtentechnologien hätten. ${ }^{78}$ Es wurde aber auch festgestellt, dass in den letzten Jahren immer mehr Dienstleistungen elektronisch angeboten werden, und eine Vertrautheit mit elektronischen Dienstleistungen könnte ein Faktor sein, der die Umsetzung von ICTs im Gerichtswesen erleichtern wird.

[39] Tabelle 3 zeigt die erwarteten Ergebnisse und Auswirkungen an den Schweizer Gerichten nach Einschätzung der befragten Personen. Je mehr «+» dieser Punkt erhielt, desto öfter wurde er von den Befragten erwähnt.

74 HIS-Programm

75 Freitas/Medeiros (2015).

76 Rosa/Teixeira/Pinto (2013).

77 Teixeira/Rêgo (2017); Andrade/Joita (2012); Sousa/Guimaraes (2014).

78 Morris/Venkatesh (2000); Sousa/Guimaraes (2014); Freitas/Medeiros (2015). 
Tabelle 3: Erwartete Ergebnisse und Auswirkungen (Schweizer Teil des Forschungsprojekts)

\begin{tabular}{lllll}
\hline Ergebnisse /Auswirkungen & $\begin{array}{l}\text { Richter } \\
(\mathrm{N}=9)\end{array}$ & $\begin{array}{l}\text { IT Manager } \\
(\mathrm{N}=5)\end{array}$ & $\begin{array}{l}\text { Justiz Manager } \\
(\mathrm{N}=8)\end{array}$ & $\begin{array}{l}\text { Summe } \\
(\mathrm{N}=22)\end{array}$ \\
\hline $\begin{array}{llll}\text { Geschwindigkeit } \\
\text { Prüfungs- und }\end{array}$ & ++++ & ++ & +++ & +++ \\
Umgestaltungsprozess (Routinen) & ++ & ++++ & +++ & +++ \\
Simultaneität & +++ & $/$ & +++ & ++ \\
Kosteneinsparungen & $/$ & ++++ & ++ & ++ \\
Mehrarbeit und Doppelerfassung & $/$ & +++ & ++ & + \\
Übermittlung von Fällen & + & +++ & + & + \\
Zugang & + & + & ++ & + \\
Mobilität & $/$ & ++ & ++ & + \\
Sicherer & $/$ & $/$ & ++ & + \\
Möglichkeit zur Verwendung von & & & & + \\
Al & $/$ & ++ & $/$ & + \\
Arbeiten am Bildschirm & $/$ & $/$ & + & + \\
Abhängigkeit von der IT & $/$ & $/$ & + & + \\
\hline
\end{tabular}

Quelle: Forschungsdaten.

Anmerkungen: ++++ steht für einen Schlüsselfaktor, der von mindestens zwei Dritteln der Befragten genannt wird; +++ von mindestens der Hälfte der Befragten erwähnt; ++ von mindestens einem Viertel der Befragten erwähnt; + mindestens einmal erwähnt; / Schlüsselfaktor nicht erwähnt.

Die Geschwindigkeit, der Prüfungs- und Umgestaltungsprozess, die Übermittlung von Fällen und der Zugang zur Innovation wurden von allen Befragten thematisiert. Geschwindigkeit ist ein von den Gerichten geplanter und gewünschter Effekt, um das Problem des Rückstaus und der Verzögerungen von Fällen zu lösen, über die in der Literatur bereits ausführlich berichtet wurde, ${ }^{79}$ wie einer der Befragten angab, wobei unter Geschwindigkeit «die Schnelligkeit der Entscheidungsfindung und der Umsetzung von Entscheidungen» verstanden wurde. Zur Anpassung von Arbeitsabläufen oder organisatorischen Routinen an das digitale oder elektronische Modell wird in den aktuellen Prozessen eine Überprüfung oder vollständige Modellerstellung erwartet. Die Übermittlung von physischen Fällen ist in einem vollständig digitalisierten Verfahren nicht notwendig, sodass sich dies auch auf die Geschwindigkeit der Leistungserbringung auswirken würde. Ebenso hätte die Bevölkerung einen besseren Zugang zu ihren Fallakten, wie ein Richter feststellt: «Es ist einfacher, auf elektronischem, digitalem Wege zum Gericht zu gelangen als durch den Postweg oder durch die Herausgabe von Dokumenten, wenn man alles elektronisch zur Verfügung hat, ist es einfacher», und statistische Auswertungen wären leichter abrufbar.

[40] Simultaneität wurde von Richtern und Justizmanagern festgehalten. Dieses erwartete Ergebnis wird als positiv bewertet. Nach Ansicht eines Richters «wird das ein enormer Vorteil sein, nicht zuletzt die Tatsache, dass verschiedene Personen alle relevanten Dokumente im selben Moment einsehen können. Wenn ein System wie dieses funktioniert, bin ich mir sicher, dass sogar

79 Sousa/Guimaraes (2014); Freitas/Medeiros (2015); Teixeira/Rêgo (2017); Weers (2016). 
die beteiligten Parteien die Akten einsehen können, während wir daran arbeiten. Wir müssen sie also nicht an die Mitarbeiter weiterleiten, damit sie sie durchsehen können, und wir haben die Unterlagen auch bei uns am Gericht.» Ausserdem sagte ein Manager: «Heutzutage haben wir die Papierunterlagen, und wenn sich die Unterlagen in einem Büro befinden, können sie nicht gleichzeitig in einem anderen Büro vorhanden sein. Wenn also alles zu jeder Zeit online verfügbar ist, kann jeder zu dem Zeitpunkt arbeiten, zu dem er arbeiten möchte.»

[41] Kosteneinsparungen, Mehrarbeit und Doppelerfassung und Mobilität wurden von Justizmanagern und IT-Managern hervorgehoben. Die Kosteneinsparung ist eine zu erwartende wirtschaftliche Auswirkung aufgrund der Reduzierung des Versands von Dokumenten per Post, des Druckens, der Einsparung von Lagerfläche für gedruckte Akten und der Reduzierung des Aktentransports innerhalb der Gerichte, Auswirkungen, die auch in früheren Studien beobachtet wurden. ${ }^{80}$ Mehrarbeit und Doppelerfassung werden mit der gleichzeitigen Verwendung physischer und elektronischer Systeme assoziiert, insbesondere bei der Umsetzung und Anpassung an das neue digitalisierte System. Allerdings wurde dies bei einer Umsetzung auch als positives Ergebnis bewertet, wie ein Befragter erwähnt: «Ich denke, der positive Effekt besteht darin, dass wir die Informationen nicht mehr als einmal in unsere Systeme eingeben müssen, denn heutzutage muss jedes Gericht alle Informationen in sein System eingeben, und das andere Gericht muss dies erneut tun.» Mobilität wird mit der Möglichkeit der Nutzung mobiler Geräte durch die Gerichte assoziiert. Wie ein Manager betont: «Wir haben vom PC zum Notebook gewechselt, das ganze Justizwesen verändern wir, und dadurch verfügen wir über mehr Mobilität, mehr Möglichkeiten für mehr Mobilität in der Zukunft.»

[42] Sicherheit, Arbeit am Bildschirm und die Abhängigkeit von der IT wurden nur von Justizmanagern festgehalten. Sie sind der Meinung, dass mit der Umsetzung der Innovation die Sicherheit erhöht wird, sodass das Risiko eines Dokumentenverlusts bei der digitalen Übertragung von Dokumenten sinkt. Es wurde auch erwähnt, dass individuelle Umstellungen erforderlich sein werden, um lange Stunden am Computerbildschirm lesen und arbeiten zu können. Eine weitere Sorge war die zunehmende Abhängigkeit von der IT. Diese Abhängigkeit ist bei der Umsetzung von ICTs in anderen Ländern aufgrund der ununterbrochenen Verfügbarkeit von Informationssystemen, deren Sicherheit, Compliance, Support und der damit verbundenen erhöhten Verantwortung im IT-Bereich zu beobachten. ${ }^{81}$

[43] Die Möglichkeit, AI zu benutzen, wurde nur von IT-Managern ins Spiel gebracht. Interessant zu beobachten ist, dass AI-basierte Computer-Tools in verschiedenen Gerichten verfügbar gemacht werden, z.B. das Victor-Projekt und das Socrates-Projekt an brasilianischen Obergerichten. Ein weiteres interessantes Beispiel sind Online-Tools zur Streitschlichtung durch AI an australischen Gerichten. ${ }^{82}$

[44] Es ist wichtig zu betonen, dass sich das Projekt Justitia 4.0 noch im Frühstadium befindet, dies ist eine Einschränkung dieser Studie. Gemäss früheren Studien über die Umsetzung von Innovationen im Justizwesen ergeben sich negative Auswirkungen auf diese Erfahrungen in den Phasen nach der Umsetzung, die in dieser Untersuchung nicht erwähnt wurden und die vom Team, welches diese Innovation im Schweizer Justizwesen umsetzt, berücksichtigt werden kön-

80 Sousa/Guimaraes (2014); Freitas/Medeiros(2015); Teixeira/Rêgo (2017); Prescott (2017).

81 Tarouco/Graeml (2011); Sousa/Guimaraes (2014).

82 ZELEZNIKOW (2017). 
nen. Wie zum Beispiel: (i) obwohl Kostensenkung und Geschwindigkeitssteigerung erwähnt wurden, kann es anfänglich zu einer Zunahme des Druckvolumens, einer Abnahme der Geschwindigkeit und einer Erhöhung der Arbeitsbelastung kommen, da es einige Zeit dauert, bis die Mitarbeiter neue Arbeitsvorgänge und digitale Dokumente akzeptiert und sich daran gewöhnt haben; ${ }^{83}$ (ii) gesundheitliche Belastungen und die Möglichkeit einer Zunahme von berufsbedingten Erkrankungen wie «[...] repetitive strain injury (RSI), work-related musculoskeletal disorders (MSDs), problems with the cervical spine, vision problems, sedentary lifestyles, absenteeism, increased stress, dissatisfaction, sadness, overwork and difficulty in disconnecting from work; ${ }^{84}$ (iii) die Bedeutung der Zusammenarbeit zwischen Gerichten und anderen staatlichen Stellen oder mit älteren Systemen; ${ }^{85}$ (iv) die Notwendigkeit nationale Normen festzulegen. ${ }^{86}$

[45] Darüber hinaus können weitere Lehren aus anderen Studien bei der Umsetzung und Implementierung nützlich sein, wie z.B: (i) die Einbeziehung von Rechtsexperten und die Zusammenarbeit mit zukünftigen Nutzern, die Einbeziehung von Feedback aus den verschiedenen Interessensgruppen mit Präsentations- und Vorführungsveranstaltungen, das Gefühl der Beteiligung und des Mitwirkens kann die Umsetzung eher erleichtern; ${ }^{87}$ (ii) die Infrastrukturplanung in Extremfällen, wie Naturkatastrophen oder menschlichen Katastrophen, ${ }^{88}$ (iii) Sicherheit, Integrität und Geheimhaltung von Daten und Informationen sind wesentliche Elemente; ${ }^{89}$ (iv) die Schaffung einer Organisationsstruktur zur Unterstützung von Innovation. ${ }^{90}$

\section{Fazit und Anmerkungen}

[46] Zur Beantwortung der Forschungsfrage «Welches sind die wichtigsten Schlüsselfaktoren, d.h. Triebkräfte und Hindernisse, bei der Einführung von E-Gerichten in der Schweiz?» wurde folgendes Ziel definiert: Beschreibung der Erfahrungen und Strategien, die bei der Umsetzung der Innovation angewandt wurden; Diskussion der relevanten Schlüsselfaktoren für die Einführung von Innovationen; Erörterung der erwarteten Ergebnisse und Auswirkungen bei der Implementierung von E-Gerichten in der Schweiz.

[47] Das Projekt Justitia 4.0 ist ein Projekt mit einer landesweiten Reichweite und hat das Potenzial, die Schweizer Justiz durch die Digitalisierung und das Angebot eines Online-Portals zu verändern. Das Projekt ist im Aufbau, weshalb diese Studie Informationen liefert, die zur Umsetzung und Implementierung dieser Innovation beitragen können.

[48] Die Ergebnisse haben die wichtigsten Schlüsselfaktoren für diese Innovation aufgezeigt, klassifiziert nach Untersuchungsebenen. Darüber hinaus wurden die von den Befragten erwarteten Hauptergebnisse vorgestellt und besprochen. Zudem wurden frühere Forschungsarbeiten zur

\footnotetext{
83 Sousa/Guimaraes (2014).

84 Sousa/Guimaraes (2014), 110.

85 Rosa/Teixeira/Pinto (2013).

86 TeIXeIra/RÊgo (2017).

87 Weers (2016); Rosa/Teixeira/Pinto (2013).

88 Rosa/Teixeira/Pinto (2013).

89 Rosa/Teixeira/Pinto (2013).

90 Wallace (2017).
} 
Umsetzung von Innovationen an Gerichten vorgestellt, welche dazu beitragen können, Risiken zu minimieren.

[49] Die Tatsache, dass sich das Projekt noch in einer Anfangsphase befindet, stellt eine wesentliche Einschränkung der Studie dar. Für zukünftige Forschungsprojekte wird empfohlen: (i) diese Innovation in späteren Phasen zu erforschen, insbesondere nach deren Implementierung, um die erwarteten mit den tatsächlichen Auswirkungen zu vergleichen; (ii) die Forschung auf andere Kantone und Gerichte auszuweiten, welche von dieser Studie nicht erfasst wurden; (iii) international vergleichende Studien durchzuführen, um das Implementierungsverhalten in verschiedenen Ländern und Kontexten zu überprüfen.

Marcos de Moraes Sousa ist Professor am Goiano Federal Institute und Professor an der Bundesuniversität von Goias (Universidade Federal de Goiás), Brasilien.

Der Verfasser dankt Andreas Lienhard und Daniel Kettiger vom Kompetenzzentrum für Public Management der Universität Bern für die Unterstützung bei der Durchführung der Studie und beim Verfassen des Artikels. Weiter dankt er Fabian Netos für die Übersetzung des Textes vom Englischen ins Deutsche.

\section{Literaturverzeichnis}

Andrade André/Joia Luiz A., Organizational structure and ICT strategies in the Brazilian Judiciary System, Gov. Inf. Q. 29 (2012) S32-S42.

Bal Hasan Çebi/Erkan Çısıl, Industry 4.0 and competitiveness, Procedia Comput. Sci. 158 (2019) 625-631.

Bardin Lawrence, Análise de conteúdo, São Paulo 2011.

Baxter David/Schoeman Magnus/Goffin Keith, Innovation in Justice. New delivery models and better outcomes, London 2011

Bloch Carter, Measuring public innovation in the Nordic Countries: final report, Copenhagen 2010.

Borkowski Julian, Court technology in Canada, Willian Mary Bill Rights J. 12 (2004) 681-686.

BüHLER JACQues, Caseflow management - CEPEJ and Swiss Federal Supreme Court's experiences, in: Andreas Lienhard/Daniel Kettiger (Hrsg.), Innovations on European caseflow management in courts, Bern 2018.

Bundesgericht, Am Gesamtprojekt eDossier Gerichte beteiligte Kantone, https://www.bger.ch/files/live/sites/bger/files/ pdf/Justitia_4.0/Zusammenarbeitsvertrag_Uebersicht_2020_01_D.pdf (besucht am 20. August 2019).

Carlson Scott A., eDiscovery: A new approach to discovery in federal and state courts, Ill. Bar J. 9 (2007) $184-209$.

Castro Marilú Pereira/Guimaraes T. Aquino, Dimensões da inovação em organizações da justiça: proposição de um modelo teórico-metodológico, Cad. EBAPE.BR 17 (2017) 173-184.

CEPEJ, European Judicial Systems. Use of information technology in European courts in 2016 (Q62 to Q65).ders., Crystal scales of justice prize.

ders., Evaluation of the judicial systems (2016-2018 cycle): Switzerland.CoE, European convention on human rights, https://www.echr.coe.int/Documents/Convention_ENG.pdf.

Contini Francesco, Reinventing the docket, discovering the database: The divergent adoption of information technology in the Italian judicial offices, in: Marco Fabri/Philip Langbroek (Hrsg.), The challenge of change for judicial systems: Developing a public administration perspective, Utrecht 2000

Crunkilton Dhira D., Staff and client perspectives on the journey mapping online evaluation tool in a drug court program, Eval. Program Plann. 32 (2009) 119-128.

DAvis Fred D., User acceptance of information technology: System characteristics, user perceptions and behavioral impacts, Int. J. Man. Mach. Stud. 38 (1993) 475-487. 
Davis Fred D./Bagozzi Richard P./Warshaw Paul R., User acceptance of computer technology: A comparison of two theoretical models, Manage. Sci. 35 (1989) 982-1003.

Dijk Frans Van/Dumbrava Horatius, Judiciary in times of scarcity: Retrenchment and reform, Int. J. Court Adm. 5 (2013) $1-10$.

Dillon Mark/Beresford David, Electronic courts and the challenges in managing evidence: A view from inside the international criminal court, Int. J. Court Adm. 6 (2014) 29-36.

Dumoulin Laurence/Licoppe Christian, Videoconferencing, new public management, and organizational reform in the judiciary, Policy and Internet 8 (2016) 133-333.

Fernandes Ricardo Vieira Carvalho/Rule Colin/Ono Taynara Tiemi/Cardoso Gabriel Estevam Botelho, The expansion of onlne dispute resolution in Brazil, Int. J. Court Adm. 9 (2018) 20-30.

Freeman Chris/Soete Luc, A economia da inovação industrial, Campinas 2008.

Freitas Christiana Soares/Medeiros Janann Joslin, Organizational impacts of the electronic processing system of the Brazilian Superior Court of Justice, J. Inf. Syst. Technol. Manag. 12 (2015) 317-332.

Gomes Adalmir de Oliveira/Alves Simone Tiêssa/Silva Jéssica Traguetto, Effects of investment in information and communication technologies on productivity of courts in Brazil, Gov. Inf. Q. 35 (2018) 480-490.

Greacen John M., Eighteen ways courts should use technology to better serve their customer, Fam. Court Rev. 57 (2019) 515-538.

Greenwood Michael/Bockweg Gary, Insights to building a successful e-filing case management service: U.S. Federal Court experience, Int. J. Court Adm. 4 (2012) 2-10.

Guimarães Tomas A./Gomes Adalmir O./Guarido Filho Edson R., Administration of justice: An emerging research field, RAUSP-Management J. 53 (2018) 476-482.

HIS-Programm, Vision und Zielsetzungen Justitia 4.0, https://www.his-programm.ch/de/Projekte/Justitia-40/Ziele (besucht am 20. September 2018).

ders., Que signifie bacs à sable?, https://www.justitia40.ch/fr/methodologie/ (besucht am 8. Mai 2020).

Joia Luiz A., Governo eletrônico e capital intelectual nas organizações públicas, Rev. Adm. Pública 43 (2009) 1379-1405.

Katz Elihu/Levin Martin L./Hamilton Herbert, Traditions of research on the diffusion of innovation, Am. Sociol. Rev. 28 (1963) 237-252.

Kettiger Daniel/Lienhard Andreas/Langbroek Philip/Fabri Marco, Court management: A young field of public management, in: Edoardo Ongaro (Hrsg.), Public Administration in Europe: The contribution of EGPA, London 2019, 309-315.

Koch Per/Cunningham Paul/Schwabsky Nitza/Hauknes Johan, Summary and policy recommendations, Oslo 2006.

Lienhard Andreas, Performance assessment in courts - The Swiss case - Constitutional appraisal and thoughts as to its organization, Int. J. Court Adm. 6 (2014) 26-42.

Lienhard Andreas/Kettiger Daniel, Between management and the rule of law: Results of the research project «basic research into court management in Switzerland», Int. J. Court Adm. 8 (2017) 7-19.

Lienhard Andreas/Kettiger Daniel/Uster Hanspeter/Winkler Daniela, Geschäftslast sowie Aufbau- und Ablauforganisation der Gerichte und der Staatsanwaltschaft im Kanton Basel-Stadt, Schlussbericht, KPM, Bern (2015).

Magnuson Eric J./Thumma Samual A., Prospects and problems associated with technological change in appellate courts: Envisioning the appeal of the future, J. Appell. Pract. Process 15 (2014) 111-138.

McKechnie Dougal, The use of the internet by courts and the judiciary: Findings from a study trip and supplementary research, Int. J. Law Inf. Technol. 11 (2003) 109-148.

MeYer JR. Norman H., Social media and the courts: Innovative tools or dangerous fad? A practical guide for court administrators, Int. J. Court Adm. 6 (2014) 22-28.

Morris Michael G./Venkatesh Viswanath, Age differences in technology adoption decisions: Implications for a changing work force, Pers. Psychol. 53 (2000) 375-403.

Pangalos George/Salmatzidis Ioannis/Pagkalos Ioannis, Using IT to provide easier acess to cross-border legal procedures for citizens and legal professionals - Implementation of a European Payment Order E-Codex pilot, Int. J. Court Adm. 6 (2014) 43-52.

Prescott James J., Improving access to justice in state courts with platform technology, Vanderbilt Law Rev. 70 (2017) 1993-2050.

Procopiuck Mario, Information technology and time of judgment in specialized courts: What is the impact of changing from physical to electronic processing?, Gov. Inf. Q. 35 (2018) 491-501.

ReILING Dory, Technology in courts in Europe: Opinions, practices and innovations, Int. J. Court Adm. 4-20 (2012) 11. 
Rogers Everett M., Diffusion of innovations, 5. Aufl., New York 2003.

Rosa João/Teixeira Claudio/Pinto Joaquim Sousa, Risk factors in e-justice information systems, Gov. Inf. Q. 30 (2013) $241-256$.

Sousa Marcos de Moraes/Guimaraes T. A., Inovação e desempenho na administração judicial: Desvendando lacunas conceituais e metodológicas, Rev. Adm. e Inovação 11 (2014) 321-344.

ders., The adoption of innovations in Brazilian labor courts from the perspective of judges and court managers, Rev. Adm. 52 (2017) 103-113.

Stadelmann Thomas, Justizkommunikation -- alte und neue Medien, in: «Justice - Justiz - Giustizia» $2018 / 2$.

Staechelé François, Les technologies de l'information au service de la modernisation du service public de la justice en France, in: Marco Fabri/Philip Langbroek (Hrsg.), The challenge of change for judicial systems: Developing a public administration perspective, Utrecht 2000 .

Switzerland, Federal Constitution of the Swiss Confederation, https://www.admin.ch/opc/en/classified-compilation/ 19995395/index.html\#a191c (besucht am 5. Mai 2020).

Tarouco Hiury Hakim/Graeml Alexandre Reis, Governança de tecnologia da informação: Um panorama da adoção de modelos de melhores praticas por empresas brasileiras usuárias, Rev. Adm. 46 (2011) 7-18.

Teixeira Janaina Angelina/Rêgo Mariana Carolina Barbosa, Inovação no sistema judiciário com a adoção do processo judicial eletrônico em um tribunal de justiça brasileiro, J. Adm. Sci. 23 (2017) 369-384.

Velicogna Marco/Errera Antoine/Derlange Stéphane, e-Justice in France: The e-Barreau experience, Utr. Law Rev. 7 (2011) 163-187.

ders., Building e-justice in Continental Europe: The TéléRecours experience in France, Utr. Law Rev. 9 (2013) 38-59.

VRies Hanna de/Bekkers Victor/Tummers Lars, Innovation in the public sector: A systematic review and future research agenda, Public Adm. 94 (2016) 146-166.

Wallace AnNe, The impact of technology on courts, Int. J. Court Adm. 8 (2017) 2156-7964.

Warren Marilyn, Open justice in the technological age, Monarch Univ. Law Rev. 40 (2014) 45-58.

Weers Thomas, Case flow management Net-project - The practical value for civil justice in the Netherlands, Int. J. Court Adm. 8 (2016) 31-42.

Zeleznikow John, Can artificial intelligence and online disupute resolution enhance efficiency and effectiveness in courts, Int. J. Court Adm. 8 (2017) 30-45. 